\title{
TetMYB DNA Vaccine
}

National Cancer Institute

\section{Source}

National Cancer Institute. TetMYB DNA Vaccine. NCI Thesaurus. Code C153094.

A therapeutic eng ineered DNA vaccine composed of DNA sequences encoding for one or more tetanus toxoid peptides and the oncoprotein MYB, with potential immunostimulatory and antineoplastic activities. Upon intradermal administration, TetMYB is taken up and processed by dendritic cells (DCs), which present the processed antigen to the immune system. This activates cytotoxic T-lymphocytes (CTLS) and causes a CT L-mediated immune response against MYB-expressing tumor cells. MYB, an oncoprotein and transcription factor, is overexpressed in a variety of cancer types and is essential for tumor cell growth, inhibition of differentiation, and protection from apoptosis. Its expression is correlated with lower T-cell infiltration and poorer prognosis. As MYB is only weakly immunogenic, the tetanus toxoid peptides enhance the T-cell mediated immune responses against the MYB-expressing tumor cells. 